\title{
Effect of selenium supplementation on lipid profile in hemodialysis patients
}

\author{
Hamidreza Omrani ${ }^{1}$, Sima Golmohamadi ${ }^{*}$, Yahya Pasdar ${ }^{2}$, Kambiz Jasemi $^{3}$, Afshin Almasi ${ }^{4}$ \\ ${ }^{1}$ Nephrology and Urology Research Center, Kermanshah University of Medical Sciences, Kermanshah, Iran \\ ${ }^{2}$ Department of Nutrition, Kermanshah University of Medical Sciences, Kermanshah, Iran \\ ${ }^{3}$ Department of Internal Medicine, Kermanshah University of Medical Sciences, Kermanshah, Iran \\ ${ }^{4}$ Department of Biostatistics and Epidemiology, Kermanshah University of Medical Sciences, Kermanshah, Iran
}

\section{A R T I C L E I N F O}

Article Type:

Original

\section{Article History:}

Received: 14 April 2016

Accepted: 20 May 2016

Published online: 30 May 2016

\section{Keywords:}

Chronic kidney disease

Hemodialysis

Selenium

Lipid

End stage renal disease

\begin{abstract}
A B S T R A C T
Introduction: One of the major causes of mortality in chronic kidney disease (CKD) patients on hemodialysis is premature atherosclerosis. Selenium, a trace element involved in important enzymatic activities inside the body, has protective effects against lipid oxidation and inhibits cholesterol accumulation in blood vessels.

Objectives: To determine the effect of selenium supplementation on lipid profile in hemodialysis patients.

Patients and Methods: In this double-blinded randomized clinical trial which lasted for 3 months, 84 hemodialysis patients with selenium deficiency were divided into experimental group (received selenium supplementation) or control group (received placebo). Total cholesterol, low-density lipoprotein (LDL-C), high-density lipoprotein (HDL-C), blood urea nitrogen (BUN), creatinine, and selenium level were measured before and after the study. Results: Mean $( \pm$ SD) serum LDL-C level significantly increased in experimental group from $85.66( \pm 31.12)$ to $109.12( \pm 32.29) \mathrm{mg} / \mathrm{dl}(P<0.001)$. Likewise, in control group serum LDL-C significantly increased from $80.55( \pm 21.13)$ to $97.05( \pm 28.07) \mathrm{mg} / \mathrm{dl}(P<0.001)$. However, with control of LDL-C effect before and after the study, it was revealed that LDL-C change was not statistically significant $(P=0.21)$. Similarly, total cholesterol and triglyceride levels did not show significant changes before and after the study in any group.

Conclusion: Selenium supplementation had no beneficial effect on lipid profile in hemodialysis patients.
\end{abstract}

\section{Implication for health policy/practice/research/medical education:}

Since there is controversy about selenium supplementation on lipid profile in patients with hemodialysis patients, we conducted a study to determine the role of omega- 3 in such patients. Our results showed that in short-term follow-up selenium supplementation, selenium supplementation had no beneficial effect on lipid profile in hemodialysis patients.

Please cite this paper as: Omrani H, Golmohamadi S, Pasdar Y, Jasemi K, Almasi A. Effect of selenium supplementation on lipid profile in hemodialysis patients. J Renal Inj Prev. 2016;5(4):179-182. DOI: 10.15171/jrip.2016.38

\section{Introduction}

The premature atherosclerosis is one of the main causes of death among hemodialysis patients. Dyslipidemia is the main cause of atherosclerosis development in such patients $(1,2)$. Some studies have suggested that hemodialysis per se causes increased production of free radicals (3-5). It is likely that some small proteins such as immunoglobulin $\mathrm{G}$ (IgG) and complement system elements adhere to the membrane of dialysis system and cause granulocyte activation which in turn causes production of free radicals $(1,2,6)$. Hence, increased lipid peroxidation as well as antioxidant depletion could be the contributing factors in atherosclerosis in hemodialysis patients $(1,6,7)$.

Recently, the role of lipid peroxidation products has drowned widespread attention in the pathogenesis of atherosclerosis (7). Oxidative stress, as a result of excessive production of reactive oxygen species (ROS) by activated monocytes and depression of antioxidant system, exists in chronic kidney disease (CKD) patients. This oxidative stress facilitates the process of atherogenesis in CKD patients. A growing body of evidence indicates a decrease in antioxidant function for the elimination of ROS in hemodialysis patients $(1,2)$. Among the most important antioxidant defense systems of body are glutathione per- 
oxide (GSH-PX) and superoxide dismutase (SOD) enzymes which their performances require copper, zinc and selenium.

Selenium is a trace element and is an essential component of GSH-PX (Ec1.11.1.9) (8). Selenium is an important trace mineral in the human body. This nutrient is an essential part of enzymes of the body's cells against free radicals in which by participating in the structure of GSH-PX will protect them. Free radicals are formed during natural oxygen metabolism process of the body and have widespread destructive effects on the body. Among essential functions of selenium in the body which can also be cited is the role it plays in preventing both heart disease and elevated blood pressure $(9,10)$. Selenium by protecting lipid inhibits its oxidation, in particular LDL, which in turn prevents cholesterol deposition in arterial wall (11).

Chen et al (12) compared 81 hemodialysis patients with 42 healthy individuals and found out that selenium level in patients with chronic renal failure is significantly lower than in the healthy individuals, and consequently, the high prevalence of selenium deficiency and increased levels of cadmium have been observed in patients with renal failure. In another report, it was shown that serum selenium level in hemodialysis patients was significantly lower than in control group, and there was a negative correlation between selenium level and uric acid which may involve the risk of heart disease (13). Fujishima et al in a study conducted on 1041 patients, who were divided according to their levels of serum selenium into four groups, concluded that serum level of selenium has an inverse relationship with the risks of death among patients, particularly risk of death as a result of infectious disease among hemodialysis patients, and any decreased serum level of selenium can be a contributing factor in reducing the levels of safety, and it may increase the risk of death from infectious disease in hemodialysis patients (14).

Since micronutrients are important components in metabolism pathways of proteins and enzymes and have many metabolic functions, any changes in their serum level can have profound impact on other important and critical parts of the body. In view of the fact that antioxidant activity is lowered in patients with CKD, we decided to evaluate the role of selenium as an effective factor in antioxidant activity of these patients. Since such a study has never been conducted in Iran, and no effort has been made in previous studies in attempting to homogenize the two groups and eliminate potential risk factors, this research attempts to study hemodialysis patients and determine the levels of serum of selenium and its impact on patient's biochemical parameters for the purposes of reducing mortality and morbidity of cardiovascular (given that cardiovascular disease is the most common cause of mortality in these patients as taken a positive step in reducing complications in hemodialysis patients).

\section{Patients and Methods}

In this double-blinded clinical trial, the study population consisted of all patients who presented to out tertiary care center to undergo hemodialysis and has passed 6 months from hemodialysis initiation. Exclusion criteria were viral hepatitis or other infectious diseases, taking steroidal and non-steroidal anti-inflammatory drugs, taking of vitamins $\mathrm{E}$ and $\mathrm{C}$ during the two preceding months prior to the study, and having normal serum level of selenium. The sampling method was of convenience method and sample size was calculated as 42 patients in each group using selenium level and data of a previous study (15) considering confidence level of $99 \%$ and power of $95 \%$.

Before connection to hemodialysis machine, $3 \mathrm{cc}$ of venous blood was obtained and the following markers were measured: complete blood count (CBC), total cholesterol, low-density lipoprotein (LDL), high-density lipoprotein (HDL), blood urea nitrogen (BUN), creatinine, and selenium level.

The patients were randomly divided (using random number table) into experimental and control groups. Experimental group received selenium capsules for three months, while control group received placebo (starch) capsules. After three months, the above mentioned laboratory markers were measured again. After completion of hemodialysis, weight with light clothing using a weight scale with precision of $1 \mathrm{~kg}$ was measured. Height was also measured without shoes by a meter installed on the wall with precision of $1 \mathrm{~cm}$.

In cases where lipid abnormality was severe and the patients required multi-dimensional treatments (diet, supplements, and medication), necessary treatments were delivered after completion of the study under supervision of nephrologist.

\section{Ethical issues}

The research followed the tenets of the Declaration of Helsinki. Selenium and placebo were free and the results were provided for them. In cases where lipid abnormality was severe and the patients required multi-dimensional treatments (diet, supplements, and medication), necessary treatments were delivered after completion of the study under supervision of dietician and nephrologist. The study protocol was confirmed by the Ethics Committee of Kermanshah University. Written informed consent was obtained from patients. All data were kept confidential.

\section{Statistical analysis}

The data were analyzed using the SPSS software for Windows (version 20). For description, mean and standard deviation $( \pm S D)$ were used. To compare the data between experimental and control group, independent $t$ test, paired $t$ test, univariate analysis of variance, Wilcoxon signed rank test, and Mann-Whitney-U test were applied. Significant level of $P$ was set at 0.05 .

\section{Results}

Seventy-four patients completed the study (36 in experimental group and 38 in control group). Mean $( \pm S D)$ age of experimental and control groups was $58.03( \pm 13.04)$ and $60.09( \pm 14.27)$ years, respectively $(P=0.524)$. There were 
18 males $(47.3 \%)$ in experimental group and 17 males $(47.2 \%)$ in control group $(P=0.642)$. Table 1 presents the baseline variables studied in both groups. As shown, except for selenium level and BUN, there was no significant difference between the two groups at baseline.

Mean $( \pm S D)$ serum LDL-C level significantly increased in experimental group from $85.66( \pm 31.12)$ to 109.12 $( \pm 32.29) \mathrm{mg} / \mathrm{dl}(P<0.001)$. Likewise, in control group LDL-C significantly increased from $80.55( \pm 21.13)$ to $97.05( \pm 28.07)(P<0.001)$. However, with control of LDL$C$ level before the study, it was revealed that LDL-C difference was not significant $(P=0.21)$. Total cholesterol difference did not show significant change before and after the study in any group. With control of baseline value, it did not have significant change $(P=0.95)$. Triglyceride level significantly decreased after the study in both groups $(P<0.001)$. After controlling of the baseline value of triglyceride, it was revealed that there was no significant difference between two groups $(P=0.37$; Table 2$)$.

Table 1. Comparison of baseline characteristics between experimental (selenium supplementation) and control (placebo) groups

\begin{tabular}{lccc}
\hline & Experimental & Control & $\boldsymbol{P}$ \\
\hline BMI, kg/m² & $24.18( \pm 3.86)$ & $23.35( \pm 4.58)$ & 0.40 \\
$\mathrm{SBP}, \mathrm{mm} \mathrm{Hg}$ & $140.69( \pm 24.78)$ & $140.79( \pm 20.18)$ & 0.98 \\
DBP, $\mathrm{mm} \mathrm{Hg}$ & $83.17( \pm 16.72)$ & $86.18( \pm 11.99)$ & 0.37 \\
Total cholesterol, mg/dl & $163.11( \pm 58.12)$ & $148.29( \pm 32.87)$ & 0.17 \\
LDL-C, mg/dl & $85.66( \pm 31.12)$ & $80.55( \pm 21.13)$ & 0.41 \\
HDL-C, mg/dl & $37.26( \pm 17.41)$ & $33.95( \pm 8.37)$ & 0.78 \\
Triglyceride, mg/dl & $153.22( \pm 78.77)$ & $145.32( \pm 73.2)$ & 0.70 \\
Selenium & $34.33( \pm 12.66)$ & $44.05( \pm 26.17)$ & 0.04 \\
BUN, mg/dl & $123.97( \pm 38.11)$ & $104.58( \pm 37.56)$ & 0.03 \\
Creatinine, $\mathrm{mg} / \mathrm{dl}$ & $7.55( \pm 2.51)$ & $7.52( \pm 2.33)$ & 0.95 \\
\hline
\end{tabular}

Abbreviations: BMI, body mass index; SBP, systolic blood pressure; DBP, diastolic blood pressure; LDL, low-density lipoprotein; HDL, highdensity lipoprotein; BUN, blood urea nitrogen

All data are expressed as mean ( \pm standard deviation).

Table 2. Comparison of studied variables between experimental (selenium supplementation) and control (placebo) groups after three months

\begin{tabular}{lccc}
\hline & Experimental & Control & $\boldsymbol{P}$ \\
\hline $\mathrm{BMI}, \mathrm{kg} / \mathrm{m}^{2}$ & $24.21( \pm 3.85)$ & $23.35( \pm 4.58)$ & 0.45 \\
$\mathrm{SBP}, \mathrm{mm} \mathrm{Hg}$ & $141.97( \pm 21.54)$ & $144.08( \pm 19.79)$ & 0.40 \\
$\mathrm{DBP}, \mathrm{mm} \mathrm{Hg}$ & $85.03( \pm 11.79)$ & $85.50( \pm 10.88)$ & 0.24 \\
Total cholesterol, mg/dl & $160.47( \pm 44.08)$ & $151.79( \pm 27.18)$ & 0.95 \\
LDL-C, mg/dl & $109.12( \pm 32.29)$ & $97.05( \pm 28.07)$ & 0.21 \\
HDL-C, mg/dl & $36.11( \pm 5.80)$ & $36.34( \pm 8.65)$ & 0.23 \\
Triglyceride, mg/dl & $113.94( \pm 82.08)$ & $98.27( \pm 65.36)$ & 0.73 \\
Selenium & $180.97( \pm 40.60)$ & $53.63( \pm 36.72)$ & $<0.001$ \\
BUN, mg/dl & $114.08( \pm 31.43)$ & $99.62( \pm 33.52)$ & 0.24 \\
Creatinine, mg/dl & $10.14( \pm 14.01)$ & $8.57( \pm 2.02)$ & 0.23 \\
\hline
\end{tabular}

Abbreviations: BMI, body mass index; SBP, systolic blood pressure; DBP, diastolic blood pressure; LDL, low-density lipoprotein; HDL, highdensity lipoprotein; BUN, blood urea nitrogen

All data are expressed as mean ( \pm standard deviation).

\section{Discussion}

We studied the effects of selenium supplementation in hemodialysis patients who suffered from low serum levels of selenium. We observed a significant difference of serum selenium among the patients who used selenium and the group that did not receive any selenium. Therefore it could be concluded that in hemodialysis patients whose levels of selenium is low, supplemental selenium raise their serum selenium level. This is comparable to a former study which reported that intravenous supplemental selenium (400 mg) two times per week for two months improved the oxygen radical scavenger system and increases selenium concentrations in plasma and erythrocytes and the activity of selenium dependent glutathione peroxidase (16). Pakfetrat et al (15) studied 35 hemodialysis patients, 34 continuous ambulatory peritoneal dialysis (CAPD) patients in addition to a healthy group for a period of more than 3 months. The results suggest that selenium level in hemodialysis patients was significantly higher compared to CAPD patients, and in comparison to the healthy group the levels are significantly lower in these patient groups. Therefore, CAPD patients are at higher risk of effects of lower selenium levels than the hemodialysis patients. Also, Chen et al (12) in a study conducted on 81 hemodialysis patients and 42 healthy individuals found out that the levels of selenium in patients with chronic renal failure are significantly lower than in healthy individuals which are consistent with the findings of our research. However, given the differences in the selenium levels between the two groups, there was no significant difference between their levels of LDL-C.

Also, in another study conducted by Martí del Moral et al (13) on 117 hemodialysis patients for a period of two years, they found that the levels of selenium in dialysis patients were significantly lower than selenium levels in the individuals in the controlled group, and it could be an effective element in risk factors for atherosclerosis. Since the increase in lipids leads to atherosclerosis in hemodialysis patients, thus any association between selenium and lipid lowering offsetting a reduction of LDL-C and triglycerides have not been found in our study. Also, consumption of other antioxidants such as vitamins $\mathrm{C}$ and $\mathrm{E}$ and their effects on lipid oxidation levels could be another factor in cause changes in blood lipid levels in hemodialysis patients. Likewise, in a study conducted by TacconeGallucci et al on 103 hemodialysis patients and 39 healthy individuals concluded that serum concentration of selenium levels in hemodialysis patients who are treated with statins are higher than that in hemodialysis patients who were not treated with statins (11).

\section{Conclusion}

Selenium supplementation in hemodialysis patients does not help reducing the harmful blood lipids levels as well as lowering LDL-C and cholesterol. And yet, contradictory results of other research studies show that it is imperative to conduct further similar studies on the effects of control of diet of patients in bigger population. 


\section{Limitations of the study}

In this project, there were some limitations including 1) limitation in the number of patients interested in participating in the study and taking selenium, 2) migration of the patients, and 3) the probability of mortality at older ages among patients undergoing chronic hemodialysis or lack of regular drug use in older age. Therefore, to avoid such limitations, larger sample should be considered before starting the study.

\section{Suggestions}

The research team, in order to achieve the best results regarding the effects of selenium functions on the items discussed in the text, offers the following suggestions: 1) To perform studies with larger sample size, and 2) Long-term follow-up of patients in order to review and confirm or reject the effects of selenium on the studied items.

\section{Acknowledgments}

We thank Mr. Masoud Ahmadi-Masoul and Ms. Neda Saedpanah, the staff nurse of the hemodialysis center and also Imam Reza hospital for their kind contribution to this study.

\section{Authors' contribution}

All authors contributed to design of the research and all authors have read, revised, and approved the final manuscript.

\section{Conflicts of interest}

The authors declared no competing interests.

\section{Ethical considerations}

Ethical issues (including plagiarism, misconduct, data fabrication, falsification, double publication or submission, redundancy) have been completely observed by the authors.

\section{Funding/Support}

This manuscript extracted from MSc thesis 92236 that supported financially by deputy of research of Kermanshah University of Medical Science. The authors are grateful to all who helped in conducting the present Research.

\section{References}

1. Stosovic M, Stanojevic M, Simic-Ogrizovic S, Jovanovic D, Djukanovic L. The predictivevalue of anthropometric parameters on mortality in haemodialysis patients. Nephrol Dial Transplant. 2011;26:1367-74. doi: 10.1093/ndt/gfq497.

2. Pecoits-Filho R, Lindholm B, Stenvinkel P. The malnutrition, inflammation, and atherosclerosis (MIA) syndrome - the heart of the matter. Nephrol Dial Transplant. 2002;17:2831. doi: 10.1093/ndt/17.suppl_11.28.

3. Dukkipati R, Kopple JD. Causes and prevention of proteinenergy wasting in chronic kidney failure. Semin Nephrol 2009;29:39-49. doi: 10.1016/j.semnephrol.2008.10.006.

4. Fouque D, Kalantar-Zadeh K, Kopple J, Cano N, Chauveau $\mathrm{P}$, Cuppari L, et al. A proposed nomenclature and diagnostic criteria for protein-energy wasting in acute and chronic kidney disease. Kidney Int. 2008;73:391-8. doi: 10.1038/ sj.ki.5002585.

5. Kalantar-Zadeh K, Ikizler TA, Block G, Avram MM, Kopple JD. Malnutrition-inflammation complex syndrome in dialysis patients: causes and consequences. Am J Kidney Dis. 2003;42:864-81. doi: 10.1016/j.ajkd.2003.07.016.

6. Pifer TB, McCullough KP, Port FK, Goodkin DA, Maroni BJ, Held PJ, et al. Mortality risk in hemodialysis patients and changes in nutritional indicators: DOPPS. Kidney Int. 2002;62:2238-45.

7. Combe C, McCullough KP, Asano Y, Ginsberg N, Maroni BJ, Pifer TB. Kidney Disease Out-comes Quality Initiative (K/DOQI) and the Dialysis Outcomes and Practice Patterns Study (DOPPS): nutrition guidelines, indicators, and practices. Am J Kidney Dis. 2004;44:39-46. doi: 10.1053/j. ajkd.2004.08.010.

8. Knight GD, Tyznik WJ. The effect of dietary selenium on hurmoral immunocompetence of ponies. J Anim Sci. 1990; 68:1311-17.

9. Russo MW, Murray SC, Wurzelmann JL, Woosley JT, Sandler RS. Plasma selenium levels and the risk of colorectal adenomas. Nutr Cancer. 1997;28:125-9. doi: 10.1080/01635589709514563.

10. Psathakis D, Wedemeyer N, Oevermann E, Krug F, Siegers $\mathrm{CP}$, Bruch HP. Blood selenium and glutathione peroxidase status in patients with colorectal cancer. Dis Colon Rectum. 1998;41:328-335. doi: 10.1007/BF02237487.

11. Taccone-Gallucci M, Noce A, Bertucci P, Fabbri C, Manca-di-Villahermosa S, Della-Rovere FR, et al. Chronic treatment with statins increases the availability of selenium in the antioxidant defence systems of hemodialysis patients. J Trace Elem Med Biol. 2010;24:27-30. doi: 10.1016/j. jtemb.2009.06.005.

12. Chen B, Lamberts LV, Behets GJ, Zhao T, Zhou M, Liu G, et al. Selenium, lead, and cadmium levels in renal failure patients in China. Biol Trace Elem Res. 2009;131:1-12. doi: 10.1007/s12011-009-8340-y.

13. Martí del Moral L, Agil A, Navarro-Alarcón M, LópezGa de la Serrana H, Palomares-Bayo M, Oliveras-López MJ. Altered serum selenium and uric acid levels and dyslipidemia in hemodialysis patients could be associated with enhanced cardiovascular risk. Biol Trace Elem Res 2011; 144: 496-503. doi:10.1007/s12011-011-9152-4.

14. Fujishima Y, Ohsawa M, Itai K, Kato K, Tanno K, Turin TC, et al. Serum selenium levels are inversely associated with death risk among hemodialysis patients. Nephrol Dial Transplant 2011; 26: 3331-8. doi: 10.1093/ndt/gfq859.

15. Pakfetrat M, Malekmakan L, Hasheminasab M. Diminished selenium levels in hemodialysis and continuous ambulatory peritoneal dialysis patients. Biol Trace Elem Res 2010; 137: 335-9. doi: 10.1007/s12011-009-8588-2

16. Koenig JS, Fischer M, Bulant E, Tiran B, Elmadfa I, Druml W. Antioxidant status in patients on chronic hemodialysis therapy: impact of parenteral selenium supplementation. Wien KlinWochenschr 1997;109:13-9.

Copyright (C) 2016 The Author(s); Published by Nickan Research Institute. This is an open-access article distributed under the terms of the Creative Commons Attribution License (http://creativecommons.org/licenses/by/4.0), which permits unrestricted use, distribution, and reproduction in any medium, provided the original work is properly cited. 